\title{
Nematic Order by Disorder in Spin-2 Bose-Einstein Condensates
}

\section{Citation}

Turner, Ari M., Ryan Barnett, Eugene Demler, and Ashvin Vishwanath. 2007. “Nematic Order by Disorder in Spin-2 Bose-Einstein Condensates." Physical Review Letters 98 (19) (May 11). doi:10.1103/physrevlett.98.190404.

\section{Published Version}

doi:10.1103/PhysRevLett.98.190404

\section{Permanent link}

http://nrs.harvard.edu/urn-3:HUL.InstRepos:27891676

\section{Terms of Use}

This article was downloaded from Harvard University's DASH repository, and is made available under the terms and conditions applicable to Other Posted Material, as set forth at http:// nrs.harvard.edu/urn-3:HUL.InstRepos:dash.current.terms-of-use\#LAA

\section{Share Your Story}

The Harvard community has made this article openly available.

Please share how this access benefits you. Submit a story.

Accessibility 


\title{
Nematic Order by Disorder in Spin-2 Bose-Einstein Condensates
}

\author{
Ari M. Turner, ${ }^{1}$ Ryan Barnett, ${ }^{2}$ Eugene Demler, ${ }^{1}$ and Ashvin Vishwanath ${ }^{3}$ \\ ${ }^{1}$ Department of Physics, Harvard University, Cambridge, Massachusetts 02138, USA \\ ${ }^{2}$ Department of Physics, California Institute of Technology, MC 114-36, Pasadena, California 91125,USA \\ ${ }^{3}$ Department of Physics, University of California, Berkeley, California 94720, USA
}

(Received 6 February 2007; published 11 May 2007)

\begin{abstract}
We show that quantum and thermal fluctuations in spin-2 Bose-Einstein condensates lift the accidental degeneracy of the mean-field phase diagram. Fluctuations select the uniaxial (square biaxial) nematic state for scattering lengths $a_{4}>a_{2}\left(a_{4}<a_{2}\right)$. Paradoxically, the order is stronger at higher temperatures. For spin- $2{ }^{87} \mathrm{Rb}$ and ${ }^{23} \mathrm{Na}$, a continuous Ising-type transition is predicted on raising the magnetic field, from a fluctuation stabilized uniaxial state to a field stabilized square biaxial order state. This is a promising experimental system to realize the "order-by-disorder" phenomenon.
\end{abstract}

DOI: 10.1103/PhysRevLett.98.190404

The ground-state properties as well as the dynamics of spinor Bose-Einstein condensates have been the subject of many experimental studies over the past decade (see, for instance, [1-5]). The spin degree of freedom introduces a rich variety of spin ordered superfluids [6-8] and the interaction between spin order and superfluidity has several interesting consequences, for example, topological defects of the magnetic texture that trap vorticity [9]. The precise nature of the spin order realized in the ground state depends on the spin-dependent two body interaction between atoms, parametrized by the scattering length in different total-spin channels. Usually, a specification of these scattering lengths uniquely fixes the spin configuration of the condensate. An interesting exception occurs in the case of spin-2 atoms. Here, depending on the relative values of the scattering lengths in the total-spin 0,2 , and 4 channels $\left(a_{0}, a_{2}, a_{4}\right)$, one obtains either a ferromagnetic state, with a net spin moment, a cyclic (or "tetrahedratic") state, which breaks time reversal symmetry but does not have a net moment, or a nematic state which preserves time reversal invariance. In contrast to the first two states, the nematic state requires specifying an additional parameter for its description. To visualize the parameter geometrically, the state may be represented by its "reciprocal spinor," a configuration of four points. The nematic states have the symmetry of a rectangle, and the additional parameter $\eta$ is related to the aspect ratio of this rectangle. This parameter is not fixed by the two body interactions, and a manifold of accidentally degenerate states remains at this level [10].

We show here that this degeneracy is lifted by superfluid phonons, whose velocities depend on the precise nematic state being realized. In the low temperature regime, the zero point energies of the superfluid phonons leads to an energy splitting between different nematic states. This is reminiscent of the Casimir force arising from photon zero point energies [11]; here the "force" leads to a splitting of nematic states. At higher temperatures, thermal fluctuations lift the degeneracy in the free energy via entropic effects. Remarkably, we are able to derive closed form analytic expressions for this splitting in both these limits,
PACS numbers: 05.30.Jp, 03.75.Hh, 03.75.Kk, 03.75.Mn

yielding the phase diagram shown in Fig. 1. Fluctuations select the two high symmetry states, the uniaxial nematic (with the symmetry of a line) and the square biaxial nematic (with the symmetry of the square) in the parts of the phase diagram shown. The latter is an elusive phase with a non-Abelian homotopy group, that was long sought after in liquid crystal systems, but appears naturally here. Two experimentally relevant systems, spin- $2{ }^{87} \mathrm{Rb}$ and ${ }^{23} \mathrm{Na}$, are also shown on this phase diagram. They are expected to realize the uniaxial nematic phase. Bose condensates of spin- $2{ }^{87} \mathrm{Rb}$ have been realized [2] and for the parameters of that experiment we find that the splitting between nematic states from quantum fluctuations to be of order $0.3 \mathrm{pK}$ while the free-energy splitting from thermal fluctuations can be as large as $6 \mathrm{pK}$. The latter should be readily observable in experiments with careful control over the quadratic Zeeman splitting which competes with this fluctuation mechanism and prefers a square nematic state oriented in a plane orthogonal to the field. The energy splitting per atom is much lower than the temperature, but it is the total energy that determines the ground state.

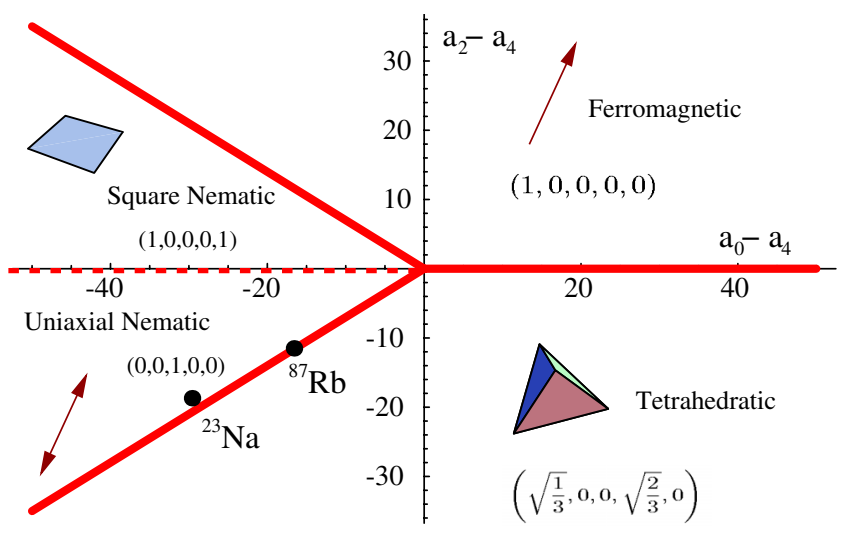

FIG. 1 (color online). The phase diagram for a spin-2 spinor condensate. The dashed line corresponds to the additional phase boundary dividing the nematic phase into two phases, a uniaxial nematic phase and a square nematic phase. 
Spin-dependent energies smaller than $T$ are known to have important effects on ground states and dynamics $[1,12]$.

An unusual feature of the fluctuation selection is that the order is stronger at higher temperatures (sufficiently far below the transition temperature), in contrast to one's intuitive expectation of thermal effects favoring disorder. Consequently, for sufficiently weak background fields, one predicts a transition on warming the system in the superfluid state, as fluctuation induced ordering overpowers the external field. Both the quantum and the thermal effects are expected to be stronger in the ${ }^{23} \mathrm{Na}$. While such "order-bydisorder" mechanisms have been widely discussed in the theory of frustrated magnetism $[13,14]$, we are not aware of an unambiguous manifestation of this effect in experiments. The spin- 2 condensates seem like a promising system to observe this intriguing effect.

Mean-field analysis. - In the dilute limit, spin-2 bosons will interact with the pair potential $V\left(\mathbf{r}_{1}-\mathbf{r}_{2}\right)=\delta\left(\mathbf{r}_{1}-\right.$ $\left.\mathbf{r}_{2}\right)\left(g_{0} \mathcal{P}_{0}+g_{2} \mathcal{P}_{2}+g_{4} \mathcal{P}_{4}\right)$ where $g_{F}=4 \pi \hbar^{2} a_{F} / m$ and $\mathcal{P}_{F}$ projects into the total-spin $F$ state. Such a pair potential leads to the interaction Hamiltonian which can be conveniently expressed as

$$
\mathcal{H}_{\text {int }}=\int d^{3} r: \frac{\alpha}{2}\left(\psi^{\dagger} \psi\right)^{2}+\frac{\beta}{2}\left|\psi^{\dagger} \mathbf{F} \psi\right|^{2}+\frac{\gamma}{2}\left|\psi^{\dagger} \psi_{t}\right|^{2}:
$$

where $\psi$ is a five component vector operator whose component $\psi_{m}(\mathbf{r})$ destroys a boson in spin state $F_{z}=m$ at position $\mathbf{r}$. Here, $\psi_{t}$ is the time reversal of $\psi$, namely, $\left(\psi_{-2}^{\dagger},-\psi_{-1}^{\dagger}, \psi_{0}^{\dagger},-\psi_{1}^{\dagger}, \psi_{2}^{\dagger}\right)$. These parameters are related to the original ones by $\alpha=\left(3 g_{4}+4 g_{2}\right) / 7, \beta=-\left(g_{2}-\right.$ $\left.g_{4}\right) / 7, \gamma=\frac{1}{5}\left(g_{0}-g_{4}\right)-\frac{2}{7}\left(g_{2}-g_{4}\right)$. The total Hamiltonian is then $\mathcal{H}=\mathcal{H}_{0}+\mathcal{H}_{\text {int }}$, where the free part is $\mathcal{H}_{0}=\int d^{3} r\left(\hbar^{2} / 2 m\right)|\nabla \psi|^{2}-\mu\left(\psi^{\dagger} \psi\right)$.

The mean-field phase diagram for such a model is shown in Fig. 1 [8]. The various extrema correspond to the ferromagnetic, nematic, and tetrahedratic phases. We will focus on the region $(\gamma<4 \beta, 0)$ where the nematic phase is stabilized. The general nematic state, up to an overall phase, is $\phi_{\eta}=\left(\frac{\sin \eta}{\sqrt{2}}, 0, \cos \eta, 0, \frac{\sin \eta}{\sqrt{2}}\right)$ and requires specification of the additional parameter $\eta$. As $\eta$ is varied, the rectangle moves through each of the three planes $x y, y z, x z$. $\eta=\frac{n \pi}{3}$ corresponds to a uniaxial along an axis, while $\eta=$ $\left(\frac{n}{3}-\frac{1}{2}\right) \pi$ corresponds to a square in one of the coordinate planes. At this level, the different nematic states are degenerate but, as demonstrated below, fluctuations will remove this accidental degeneracy in the nematic phase, producing the dashed phase boundary at $a_{2}=a_{4}(\beta=0)$. Along this phase boundary, however, the Hamiltonian possesses $\mathrm{SO}(5)$ symmetry and hence the various nematic phases are exactly degenerate. The mean-field energy density in the nematic phase is $E_{0} / V=-\mu^{2} / 2(\alpha+\gamma)$, while the chemical potential itself is given by $\mu=(\alpha+\gamma) n_{0}$, where $n_{0}$ is the atom density. We now analyze the harmonic fluctuations about the different nematic ground states.

Fluctuations. - We follow the standard Bogoliubov theory of the weakly interacting Bose gas to derive the spec- trum of fluctuations. Consider decomposing the field operator into a dominant $c$-number piece, the mean-field expectation value, and a smaller fluctuation piece $\psi(r)=$ $\sqrt{n_{0}} \phi_{\eta}+\delta \Psi(r)$. We expand the Hamiltonian to quadratic order in the fluctuations (linear terms vanish on choosing the mean-field solution) and diagonalize the resulting Bogoliubov Hamiltonian $\mathcal{H}_{B}$. This is aided by defining new canonical bosons: $b_{1}=-\frac{i}{\sqrt{2}}\left(\delta \Psi_{1}+\delta \Psi_{-1}\right), b_{2}=$ $-\frac{1}{\sqrt{2}}\left(\delta \Psi_{1}-\delta \Psi_{-1}\right), b_{3}=\frac{i}{\sqrt{2}}\left(\delta \Psi_{2}-\delta \Psi_{-2}\right)$, and $f_{ \pm}=$ $\frac{1}{\sqrt{2}} \delta \Psi_{0} \pm \frac{i}{2}\left(\delta \Psi_{2}+\delta \Psi_{-2}\right)$. Further, we define $b_{4}=p=$ $\frac{1}{\sqrt{2}}\left(e^{i \eta} f_{+}+e^{i \eta} f_{-}\right)$and $b_{5}=q=\frac{1}{\sqrt{2}}\left(-i e^{i \eta} f_{+}+i e^{i \eta} f_{-}\right)$. The Bogoliubov Hamiltonian assumes a particularly simple form in these variables. Define velocities $v_{j}$ via $m v_{j}^{2}=$ $\left[(2 \beta-\gamma) n_{0}-2 \beta n_{0} \cos \left(2 \eta+\frac{2 \pi j}{3}\right)\right](j=1,2,3)$, or

$$
v_{j}^{2}=\frac{4 \pi n_{0} \hbar^{2}}{5 m^{2}}\left[a_{4}-a_{0}+\frac{10}{7}\left(a_{2}-a_{4}\right) \cos \left(2 \eta+\frac{4 \pi}{3} j\right)\right] .
$$

Also let $m v_{4}^{2}=(\alpha+\gamma) n_{0}, m v_{5}^{2}=-\gamma n_{0}$. Then we have

$$
\begin{aligned}
\mathcal{H}_{B}= & \sum_{\mathbf{k}} \sum_{j=1}^{5}\left[m v_{j}^{2}+\frac{\hbar^{2} \mathbf{k}^{2}}{2 m}\right] b_{j \mathbf{k}}^{\dagger} b_{j \mathbf{k}} \\
& -\frac{1}{2} m v_{j}^{2}\left(b_{j \mathbf{k}}^{\dagger} b_{j-\mathbf{k}}^{\dagger}+b_{j \mathbf{k}} b_{j-\mathbf{k}}\right) .
\end{aligned}
$$

We see that these modes are completely decoupled and readily diagonalized by the Bogoliubov transformation, having spectra of the form $\omega_{j}=k \sqrt{v_{j}^{2}+\hbar^{2} k^{2} / 4 m^{2}}$. Physically, for $1 \leq j \leq 3$, the $b_{j}$ modes are connected to spin rotations about the three coordinate axes. The $p$ boson generates phase fluctuations of the condensate since the associated wave speed $v_{4}$ involves the compressibility $n_{0}(\alpha+\gamma)$. Finally the $q$ mode corresponds to fluctuations of the $\eta$. Note, in the long wavelength limit all these modes have a linear dispersion. However, for a generic nematic superfluid, only the first four are Goldstone modes of the broken spin and phase symmetry; the fifth will actually acquire a gap once the degeneracy between the different $\eta$ configurations is removed. The contribution of these modes to the free energy is readily calculated. However, only the dispersion of the spin modes depends on the nematic parameter $\eta$ and will be responsible for lifting the accidental degeneracy. The contribution from these modes $\Delta F(\eta) / V=T \sum_{n=1}^{3} \int\left[d^{3} \mathbf{k} /(2 \pi)^{3}\right] \times$ $\log \left\{2 \sinh \left[\hbar \omega_{n}(k) / 2 T\right]\right\}$, where $V$ is the volume, will be discussed below.

Order by disorder. - Before evaluating the free energy, we make some general observations arising from symmetry. For this purpose the nematic region of the phase diagram is best described in terms of the variables $\left(a_{0}-a_{4}\right)$, the $x$ axis in Fig. 1 , and the ratio $\sigma=(10 / 7) \times$ $\left[\left(a_{4}-a_{2}\right) /\left(a_{0}-a_{4}\right)\right]$. The nematic region corresponds to $\left(a_{0}-a_{4}\right)<0$ and $|\sigma|<1$. Equation (2) for the velocities shows that the degeneracy remains unresolved along the 
line $\sigma=0$, which is also the phase boundary for the full Hamiltonian due to the $\mathrm{SO}(5)$ symmetry. The symmetry breaking grows on moving away from this axis, but has opposite effects on either side of this line. Formally, the transformation $\sigma \rightarrow-\sigma, \eta \rightarrow \eta+\pi / 2$ is an approximate symmetry.

At zero temperature, the free energy reduces to the contribution to the ground-state energy for different nematic states from the zero point motion of the harmonic modes. Remarkably, this energy splitting $\Delta E(\eta)$ may be evaluated in closed form up to an overall constant [15]:

$$
\begin{aligned}
\frac{\Delta E(\eta)}{V} & =\frac{8 m^{4}}{15 \pi^{2} \hbar^{3}} \sum_{j=1}^{3} v_{j}^{5} \\
& =\frac{8 \hbar^{2}}{15 \pi^{2} m}\left[\frac{4 \pi n_{0}\left(a_{4}-a_{0}\right)}{5}\right]^{5 / 2} g_{5 / 2}(\eta, \sigma),
\end{aligned}
$$

where it is convenient to define the series of functions: $g_{m}(\eta, \sigma)=\sum_{j=1}^{3}\left[1+\sigma \cos \left(2 \eta+\frac{2 \pi j}{3}\right)\right]^{m}$. The ground state is the one with the lowest combination of velocities, and turns out to be the square biaxial state $(\eta=\pi / 2)$ for $\sigma>0$, and the uniaxial state for $\sigma<0(\eta=0)$. The latter is relevant to spin-2 ${ }^{87} \mathrm{Rb}$ and ${ }^{23} \mathrm{Na}$, which both are believed to have $\sigma \approx-1$ [17].

At finite temperatures, thermal fluctuations are much more effective at lifting this degeneracy; the nematic phase with the larger population of thermal excitations will be entropically favored. While this may be evaluated numerically, there is a broad range of temperatures $T_{c} \gg T \gg$ $\max \left(m v_{i}^{2}\right)$ where it greatly simplifies. The lower limit is a rather small scale, related to the magnetic energy and about $1 \mathrm{nK}$ for ${ }^{87} \mathrm{Rb}$ in [2]. In this limit, the linearly dispersing modes obey equipartition, and the leading $\eta$-dependent term in the free energy is [18]

$$
\begin{aligned}
\frac{\Delta F(\eta)}{V} & =-\frac{2}{3 \pi} k_{B} T \sum_{j}\left(\frac{m v_{j}}{\hbar}\right)^{3} \\
& =-\frac{2}{3 \pi} k_{B} T\left[\frac{4 \pi n_{0}\left(a_{4}-a_{0}\right)}{5}\right]^{3 / 2} g_{3 / 2}(\eta, \sigma) .
\end{aligned}
$$

On evaluating this expression, thermal fluctuations are found to lead to the same ground states as quantum fluctuations. A plot of the free-energy variation with $\eta$ for $\sigma \approx$ -1 is in the top curve of Fig. 2 .

At higher temperatures the depletion of the condensate becomes important. As the temperature is increased, the difference in free energies initially grows due to increased thermal fluctuations. Near $T_{c}$, the free-energy splitting drops back to zero, assuming a continuous transition. These features can be seen in Fig. 3, calculated provisionally for the condensate fraction which is valid for noninteracting particles in a trap, $n(T)=n_{0}\left[1-\left(T / T_{c}\right)^{3}\right]$, with the parameters of ${ }^{87} \mathrm{Rb}$ in [2].

These results may be caricatured by an effective Landau theory, where sixth order terms are generated by fluctuations. While a full discussion of all such terms is under-

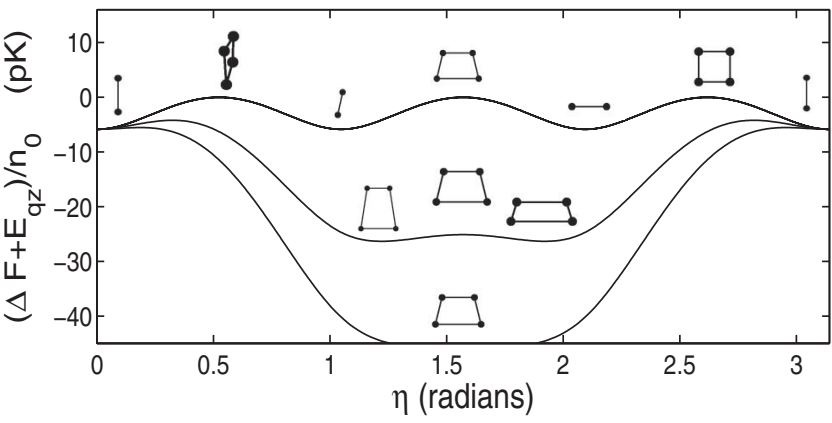

FIG. 2. Evolution of the uniaxial nematic into square biaxialnematic in a field. The free-energy, offset by a constant, is plotted in pK per atom for the conditions of Ref. [2], for $B=$ $0,20,27 \mathrm{mG}$ (top to bottom). The reciprocal spinors at the minima deform from lines into squares as the field is increased. The Ising-like symmetry breaking at the critical field corresponds to a choice of which way to compress the square.

taken elsewhere [16], we note here that writing the five component order parameter as a symmetric traceless matrix $\chi$ [19] allows for a sixth order term $\frac{32 A}{3} \operatorname{tr}\left[\chi^{3} \chi^{* 3}\right]$ in the free energy. This term, when evaluated in the nematic subspace yields $A\left|\chi_{0}\right|^{6} \cos 6 \eta$, where $\chi_{0}$ is the magnitude of the order parameter. Clearly, if $A>0$, then the free energy is minimized by the square biaxial state $\eta=$ $\pi / 2, \pi / 6,5 \pi / 6$, while if $A<0$, then the free energy is minimized by the uniaxial state $\eta=0, \pi / 3,2 \pi / 3$. This sixth order term vanishes rapidly on approaching the transition, accounting for the near degeneracy of nematic ordered states near this point. Finally, it may be recalled that biaxial nematics are rather difficult to obtain in liquid crystal systems. Within Landau theory this is explained by the presence of a cubic term in the free energy $B \operatorname{tr}\left[\chi_{R}^{3}\right]$ when the nematic order parameter is a real symmetric traceless matrix. This can be shown to favor the uniaxial state regardless of the sign of $B$. However, in the spinor condensate, the fact that the spin-2 field also carries a charge quantum number implies the absence of such a term (it is a complex symmetric matrix with phase symmetry). Hence biaxial nematics may be realized, and would naturally occur in the $\sigma>0$ part of the nematic phase region. In addition to the topological defects with nonAbelian homotopy for the general biaxial nematic, the square biaxial nematic realized here also has half superfluid vortices bound to a spin defect.

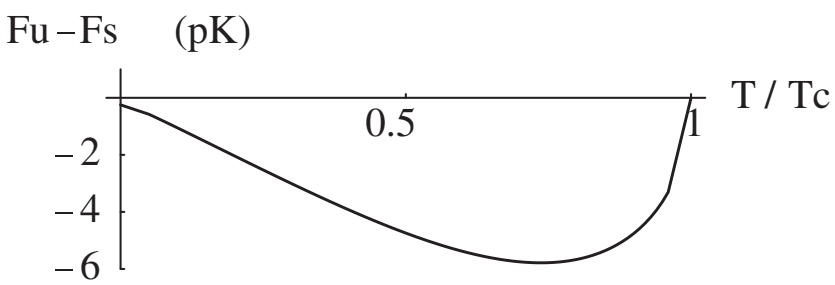

FIG. 3. Evolution of the free-energy difference between uniaxial and square biaxial-nematic states as a function of temperature with ${ }^{87} \mathrm{Rb}$ parameters. 


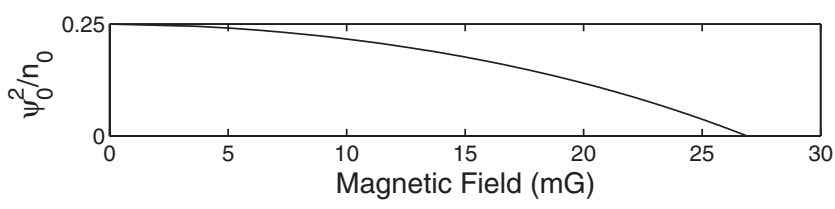

FIG. 4. Proportion of $F_{z}=0$ component as magnetic field is increased to the critical field, for rubidium at density $4 \times$ $10^{14} \mathrm{cc}^{-1}$ and $T=100 \mathrm{nK}$.

Experimental prospects. - Magnetic fields make the phase diagram more complex because the splitting due to fluctuations receives competition from the quadratic Zeeman energy $H_{q z}=-c B^{2} \int d^{3} \mathbf{x} \psi^{\dagger} F_{z}^{2} \psi$ [20], which favors the square uniaxial state. For weak fields the nematic axis drops into the $x y$ plane $\eta=\pi / 3,2 \pi / 3$ because $\left|F_{x}=0\right\rangle=\left(\sqrt{\frac{3}{8}}, 0, \frac{1}{2}, 0, \sqrt{\frac{3}{8}}\right)$ has lower $H_{q z}$ than $\left|F_{z}=0\right\rangle$. On increasing the field, the state continuously approaches the square state $\eta=\pi / 2$. In [2] a 50-50 split between atoms with $F_{z}= \pm 2$ was observed consistent with a square state; we believe this to be due to the relatively large background field reported in the experiments that overcomes the order-by-disorder effect. To observe the latter effect, a lower magnetic field as estimated below is needed. A possible way to observe the uniaxial to square state transition is by measuring the fraction of atoms with $F_{z}=0$ along a quantization axis parallel to the magnetic field as it drops from $25 \%$ for uniaxial states to zero for the square state (see Fig. 4). Magnitudes of fluctuation induced free-energy splittings are shown in Table I for ${ }^{87} \mathrm{Rb}$ and ${ }^{23} \mathrm{Na}$ both at zero temperature and at $100 \mathrm{nK}$ (roughly half the critical temperature for the density in [2]). The last column shows the critical field beyond which the quadratic Zeeman effect dominates. Since magnetic fields as low as $50 \mathrm{mG}$ are readily accessible in current experiments [5], we believe these effects are within experimental reach. The effect could also possibly be used to access the condensate temperature by measuring the critical field. In a trap, an important consideration is that the linear part of the spin wave dispersions must be above the quantization scale of the trap, $\hbar \omega_{\text {trap }}<m \max \left(v_{i}^{2}\right)$.

In conclusion, the role of fluctuations in selecting the ground-state spin structure of a spin-2 nematic condensate was studied. The phase diagram in Fig. 1 results from both quantum and thermal fluctuations. In experiments on ${ }^{23} \mathrm{Na}$ and ${ }^{87} \mathrm{Rb}$, these effects would compete against the qua-

TABLE I. Free-energy splittings at $T=0, T=100 \mathrm{nK}$, and critical fields, calculated for $n=4 \times 10^{14} \mathrm{cc}^{-1}$, as in the experiments of Ref. [2]. Though sodium's scattering lengths have a bigger spread, its critical field is lower because of its stronger quadratic Zeeman effect.

\begin{tabular}{lllc}
\hline \hline & $\Delta E$ & $\Delta F$ & $B_{c}$ \\
\hline${ }^{87} \mathrm{Rb}$ & $0.3 \mathrm{pK}$ & $6 \mathrm{pK}$ & $27 \mathrm{mG}$ \\
${ }^{23} \mathrm{Na}$ & $3 \mathrm{pK}$ & $9 \mathrm{pK}$ & $19 \mathrm{mG}$ \\
\hline \hline
\end{tabular}

dratic Zeeman term, but can predominate for sufficiently weak fields. An Ising transition marks this point, which we believe can be accessed readily in future experiments. If so, this would be one of the first experimental demonstrations of order by disorder, widely studied in the context of frustrated quantum magnetism.

We would like to thank Daniel Podolsky, Gil Refael, and Dan Stamper-Kurn for insightful discussions and Fei Zhou for alerting us to [21], where similar results are obtained. Support from the Hellman Family Fund and LBNL No. DOE-504108 (A. V.), NSF Grant No. 0132874, AFOSR and Harvard-MIT CUA (E.D. and A.M.T.), and the Sherman Fairchild Foundation (R.B.) is acknowledged.

[1] J. Stenger, S. Inouye, D. M. Stamper-Kurn, H. J. Miesner, A.P. Chikkatur, and W. Ketterle, Nature (London) 396, 345 (1998).

[2] H. Schmaljohann, M. Erhard, J. Kronjäger, M. Kottke, S. van Staa, L. Cacciapuoti, J. J. Arlt, K. Bongs, and K. Sengstock, Phys. Rev. Lett. 92, 040402 (2004).

[3] M. S. Chang, C. D. Hamley, M.D. Barrett, J. A. Sauer, K. M. Fortier, W. Zhang, L. You, and M. S. Chapman, Phys. Rev. Lett. 92, 140403 (2004).

[4] A. Griesmaier, J. Werner, S. Hensler, J. Stuhler, and T. Pfau, Phys. Rev. Lett. 94, 160401 (2005).

[5] L. E. Sadler, J. M. Higbie, S. R. Leslie, M. Vengalattore, and D. M. Stamper-Kurn, Nature (London) 443, 312 (2006).

[6] T. L. Ho, Phys. Rev. Lett. 81, 742 (1998).

[7] T. Ohmi and K. Machida, J. Phys. Soc. Jpn. 67, 1822 (1998).

[8] C. V. Ciobanu, S. K. Yip, and T. L. Ho, Phys. Rev. A 61, 033607 (2000).

[9] F. Zhou, Phys. Rev. Lett. 87, 080401 (2001).

[10] R. Barnett, A. Turner, and E. Demler, Phys. Rev. Lett. 97, 180412 (2006).

[11] H. Casimir, Proc. K. Ned. Akad. Wet. 51, 793 (1948).

[12] H. Schmaljohann, M. Erhard, J. Kronjäger, K. Sengstock, and K. Bongs, Appl. Phys. B 79, 1001 (2004).

[13] J. Villain, Z. Phys. B 33, 31 (1979).

[14] C. L. Henley, Phys. Rev. Lett. 62, 2056 (1989).

[15] In fact, this integral is divergent at large $k$ because we have left off $\eta$-independent terms which arise from the more methodical analysis [16].

[16] A. Turner, R. Barnett, E. Demler, and A. Vishwanath (to be published).

[17] $\sigma=-1$ is an exact result if the scattering is represented entirely by the spin exchange of the electrons.

[18] Potentially larger terms, proportional to $T^{5 / 2}$ and $T^{3 / 2}$ and involving the functions $g_{1}(\eta, \sigma)$, do not depend on $\eta$ due to the sum over the three spin wave modes.

[19] N. D. Mermin, Phys. Rev. A 9, 868 (1974).

[20] The linear Zeeman effect is unimportant since the total magnetization is conserved on time scales of experiments with condensates.

[21] J. L. Song, G. Semenoff, and F. Zhou, cond-mat/0702052 [Phys. Rev. Lett. (to be published)]. 